\title{
Resonance Phenomena in a Nanofluid Filled Enclosure Heated Periodically from the Side
}

\author{
Mehmet Saglam , Bugra Sarper², Orhan Aydin ${ }^{1}$ \\ ${ }^{1}$ Karadeniz Technical University \\ Department of Mechanical Engineering \\ 61080 Trabzon,Turkey \\ mehmetsaglam@ktu.edu.tr; oaydin@ktu.edu.tr \\ ${ }^{2}$ Gumushane University \\ Department of Mechanical Engineering \\ 29100 Gumushane,Turkey \\ bugrasarper@gumushane.edu.tr
}

\begin{abstract}
This study deals with natural convection of water $-\mathrm{Al}_{2} \mathrm{O}_{3}$ nanofluid in a square enclosure with periodically heated from one of its side walls. The main objective is to investigate the effect of nanoparticle volume fraction on resonance frequency of the buoyancy induced flow. ANSYS Fluent (v.15) software is used for numerical simulations and computations are carried out for three different modified Rayleigh number values between $10^{7}-10^{9}$ and five different nanoparticle volume fractions between 0.01-0.05, and different values of non-dimensional frequency between 0.01-0.04. Results are presented for the variation of the mean Nusselt number on the vertical mid-line of the enclosure with the modified Rayleigh number and nanoparticle volume fraction. It is found that the nondimensional resonance frequency is slightly decreasing with increasing nanoparticle fraction and modified Rayleigh number.
\end{abstract}

Keywords: Natural Convection, Periodic Heating, Nanofluid, Resonance Frequency.

\section{Introduction}

Natural convection in enclosures has been a widely studied problem in the literature because of its wide application areas such as electronic cooling, cooling and air-conditioning applications, building design, solar energy systems and nuclear reactors. In order to increase the heat transfer capacity, different methods have been studied such as; geometric or thermal optimization and resonant enhancement. Lately, a new method is widely discussed regarding thermal properties of the working fluids. By adding metallic nanoparticles to the base fluid and keeping them suspended, thermal properties of the fluid, and, of course, the heat transfer capacity can be improved. Studies in the literature show that even small volume fraction of nanoparticles enhance thermal properties of the base fluid and heat transfer capacity considerably.

There have been a vast number of studies on increasing heat transfer by natural convection in enclosures for different working conditions. Also, numerous studies have addressed the use of nanofluids in natural convection in enclosures. Researchers have studied the effects of types of the nanofluids, nanoparticle volume fraction on heat transfer for various boundary conditions.

Some example studies for the resonance phenomenon of periodic natural convection in enclosures are given in the following: Lage and Bejan [1] studied periodic natural convection inside an enclosure numerically and theoretically. They investigated resonance frequency of different fluids having different values of Pr and Ra. Kwak et al. [2] studied natural convection inside a square enclosure with sinusoidally varying sidewall temperature with time. Kim et al. [3] studied resonance of natural convection inside an enclosure experimentally.

Similarly, some example studies for periodic natural convection of nanofluids in enclosures are as follows: Ghasemi and Aminossadati [4] studied natural convection inside an enclosure with discrete periodic heating condition for different nanofluids numerically. Sheremet et al. [5] investigated natural convection inside an inclined cavity filled with $\mathrm{Al}_{2} \mathrm{O}_{3}$-water nanofluid with time-sinusoidal temperature boundary condition numerically. They investigated effects of Rayleigh number, oscillation frequency, nanoparticle volume fraction and inclination angle on flow and heat transfer. 
In this study, periodic natural convection in a square enclosure filled with water- $\mathrm{Al}_{2} \mathrm{O}_{3}$ nanofluid is investigated numerically. The focus of this study is to obtain resonance frequencies of different solid volume fractions of nanoparticles for varying values of the modified Rayleigh number.

\section{Numerical Study}

The schematic of the problem studied and the heat flux profile applied are shown in Fig.1. Distilled water is used as the base fluid. One of the sidewalls of the square enclosure in length " $H$ " is heated with periodically varying dimensionless heat flux $\left(Q^{\prime \prime}\right)$ and the other sidewall is kept at $293.15 \mathrm{~K}$, while horizontal walls are assumed to be adiabatic. No slip boundary condition is applied for all wall-to-fluid boundaries. At the beginning of each numerical computation, the fluid and walls are assumed to be in thermal equilibrium. As it is seen from Fig 1., $20 \%$ of deviation from the mean heat flux is defined as the wave amplitude.
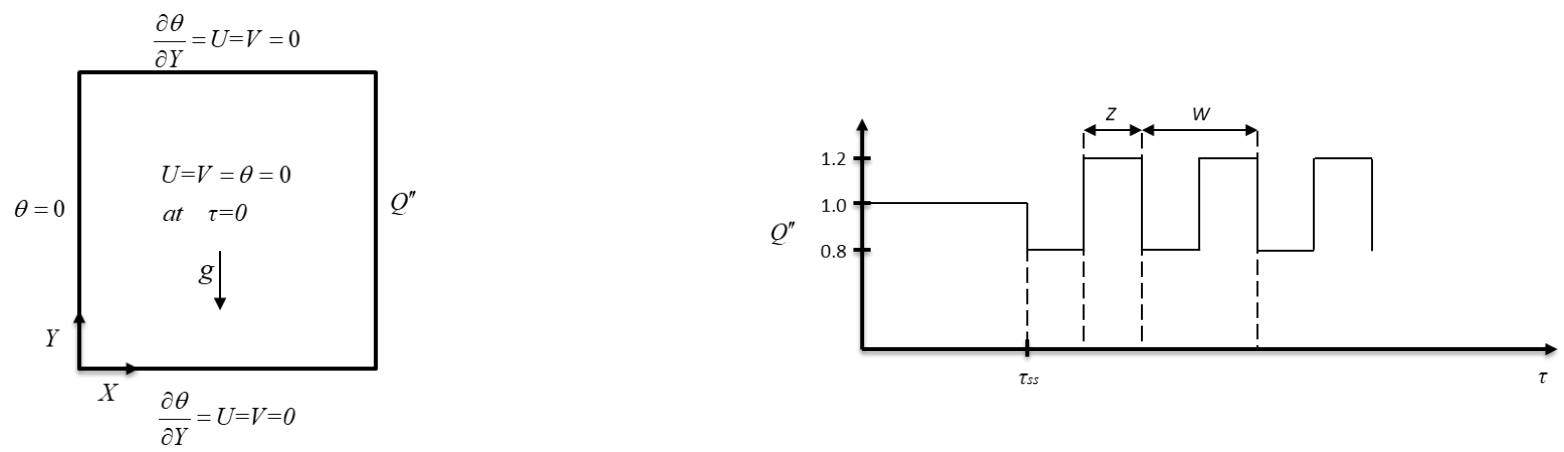

Fig. 1: The schematic of the problem and the applied heat flux.

Continuity, momentum and energy equations are solved via ANSYS Fluent software. The flow is assumed to be two dimensional, transient and laminar. Fluid properties are considered as constant except density. Density change with temperature is modelled by adopting Boussinesq approximation. For nanofluid modelling, single phase assumption is used so no slip occurs between nanoparticles and base fluid and they are in thermal equilibrium. Thermal properties of nanoparticle and distilled water are given in Table 1.

Table 1: Thermophysical properties of the materials.

\begin{tabular}{|c|c|c|c|c|}
\hline & $\boldsymbol{\rho ( \mathbf { k g } / \mathbf { m } ^ { 3 } )}$ & $\begin{array}{c}\mathbf{C}_{\mathbf{p}} \\
(\mathbf{J} / \mathbf{k g K})\end{array}$ & $\mathbf{k}(\mathbf{W} / \mathbf{m K})$ & $\boldsymbol{\beta}$ \\
\hline $\mathbf{W a t e r}$ & 998.2 & 4184.1 & 0.6034 & $0.2066 \times 10^{-3}$ \\
\hline $\mathbf{A l}_{\mathbf{2}} \mathbf{O}_{\mathbf{3}}$ & 3970 & 765 & 40 & $0.85 \times 10^{-5}$ \\
\hline
\end{tabular}

In order to define the thermal properties of nanofluid, some models which adopting base fluid $(f l)$ and nanoparticles $(n p)$ thermal properties and volume fractions $(\varphi)$ are required. Thermal properties of nanofluid is calculated by following equations given in Table 2. It should be noted that, the given numerical model is used for the dynamic viscosity although there are different experimental models in literature. 
Table 2: Applied nanofluid models.

\begin{tabular}{|c|c|}
\hline Nanofluid properties & Applied model \\
\hline Density & $\rho_{n f}=(1-\varphi) \rho_{f l}+\varphi \rho_{n p}$ \\
\hline Thermal diffusivity & $\alpha_{n f}=k_{n f} /\left(\rho C_{p}\right)_{n f}$ \\
\hline Heat capacitance & $\left(\rho C_{p}\right)_{n f}=(1-\varphi)\left(\rho C_{p}\right)_{f l}+\varphi\left(\rho C_{p}\right)_{n p}$ \\
\hline $\begin{array}{c}\text { Thermal expansion } \\
\text { coefficient }\end{array}$ & $(\rho \beta)_{n f}=(1-\varphi)(\rho \beta)_{f l}+\varphi(\rho \beta)_{n p}$ \\
\hline Dynamic viscosity & $\mu_{n f}=\mu_{f l} /(1-\varphi)^{2.5}$ \\
\hline Thermal conductivity & $k_{n f}=k_{f l}\left[\left(k_{n p}+2 k_{f l}-2 \varphi\left(k_{f l}-k_{n p}\right)\right) /\left(k_{n p}+2 k_{f l}+\varphi\left(k_{f l}-k_{n p}\right)\right)\right]$ \\
\hline
\end{tabular}

\subsection{Governing Equations}

The governing equations are non-dimensionalized with the dimensionless forms of position $(X, Y)$, velocity $(U, V)$, temperature $(\theta)$, pressure $(P)$, time $(\tau)$ parameters defined as follows:

$$
\begin{gathered}
(X, Y)=\frac{(x, y)}{H}, \quad(U, V)=\frac{(u, v) H}{\alpha_{f l}\left(R a^{*} P r\right)^{1 / 2}}, \quad \theta=\frac{T-T_{C}}{q_{m}^{\prime \prime} H}, \quad P=\frac{p H^{2}}{\rho_{n f} \alpha_{f l}^{2} R a^{*} P r}, \\
R a^{*}=\frac{g \beta_{f l} q_{m}^{\prime \prime} H^{4}}{(k \vartheta \alpha)_{f l}}, \quad P r=\frac{\vartheta_{f l}}{\alpha_{f l}}, \quad \tau=\frac{\alpha_{f l} t}{H^{2}}\left(R a^{*} P r\right)^{\frac{1}{2}}, \quad Q^{\prime \prime}=\frac{q^{\prime \prime}}{q_{m}^{\prime \prime}}
\end{gathered}
$$

where $g$ is gravitational acceleration, $\beta$ is thermal expansion coefficient, $q_{m}^{\prime \prime}$ is mean surface heat flux and $\vartheta$ is the kinematic viscosity.

The continuity, momentum and energy equations for a $2 \mathrm{D}$, transient, incompressible and laminar flow are given as follows:

$$
\begin{gathered}
\frac{\partial U}{\partial X}+\frac{\partial V}{\partial Y}=0 \\
\frac{\partial U}{\partial \tau}+U \frac{\partial U}{\partial X}+V \frac{\partial U}{\partial Y}=-\frac{\partial P}{\partial X}+\frac{\vartheta_{n f}}{\vartheta_{f l}}\left(\frac{P r}{R a^{*}}\right)^{1 / 2}\left(\frac{\partial^{2} U}{\partial X^{2}}+\frac{\partial^{2} U}{\partial Y^{2}}\right) \\
\frac{\partial V}{\partial \tau}+U \frac{\partial V}{\partial X}+V \frac{\partial V}{\partial Y}=-\frac{\partial P}{\partial Y}+\frac{\vartheta_{n f}}{\vartheta_{f l}}\left(\frac{P r}{R a^{*}}\right)^{1 / 2}\left(\frac{\partial^{2} V}{\partial X^{2}}+\frac{\partial^{2} V}{\partial Y^{2}}\right)+\frac{\beta_{n f}}{\beta_{f l}} \theta \\
\frac{\partial \theta}{\partial \tau}+U \frac{\partial \theta}{\partial X}+V \frac{\partial \theta}{\partial Y}=\frac{\alpha_{n f}}{\alpha_{f l}}\left(R a^{*} P r\right)^{-1 / 2}\left(\frac{\partial^{2} \theta}{\partial X^{2}}+\frac{\partial^{2} \theta}{\partial Y^{2}}\right)
\end{gathered}
$$

From definition of non-dimensional time, the non-dimensional period $(W)$ is obtained as 


$$
(W, Z)=(w, z)\left(\frac{\alpha_{f l}}{H^{2}}\left(R a^{*} P r\right)^{\frac{1}{2}}\right)
$$

where $w$ is the actual period. The non-dimensional frequency $(f)$ is defined as follows:

$$
W=2 Z=1 / f
$$

In order to decide whether flow is reach the resonance frequency, mean Nusselt number value on vertical midline of the enclosure is taken as reference and calculated with the definition as follows:

$$
N u_{m}=\int_{0}^{1}\left[\frac{k_{n f}}{k_{f l}} \frac{\alpha_{f l}}{\alpha_{n f}}\left(R a^{*} P r\right)^{\frac{1}{2}} U \theta-\frac{k_{n f}}{k_{f l}} \frac{\partial \theta}{\partial X}\right]_{X=1 / 2} \partial Y
$$

\subsection{Numerical Method}

Numerical study is performed by using ANSYS Fluent software. The pressure based solver is employed. For pressurevelocity coupling, SIMPLE algorithm is used. Discretization of momentum and energy equations is performed by second order upwind scheme and pressure interpolation is provided by PRESTO scheme. For transient calculations, second order implicit method is used. The solutions for all parameters have two steps as it can be seen from Fig.1. First, steady state solution is performed with mean surface heat flux for defined parameters. After steady state solution is converged, second step is started by implementing the steady state solution results as the initial condition for transient case. For each parameter, transient solutions are continued at least 4 periods of time until oscillations become regular. In order to obtain more accurate results, a non-uniform grid of $151 \times 151$ is used. Denser grids are implemented near the walls. For transient solutions, time step is taken as 0.1 second. For both steady state and transient solutions, convergence criteria is taken as $10^{-6}$. The validation of the numerical model is performed by comparing the present study with the results of Lage and Bejan [1] for $R a^{*}=10^{8}$ and $\operatorname{Pr}=7$. The comparison of the results is presented in Fig.2. As it can be seen from the figure, the results agree very well with those of Ref. [1].

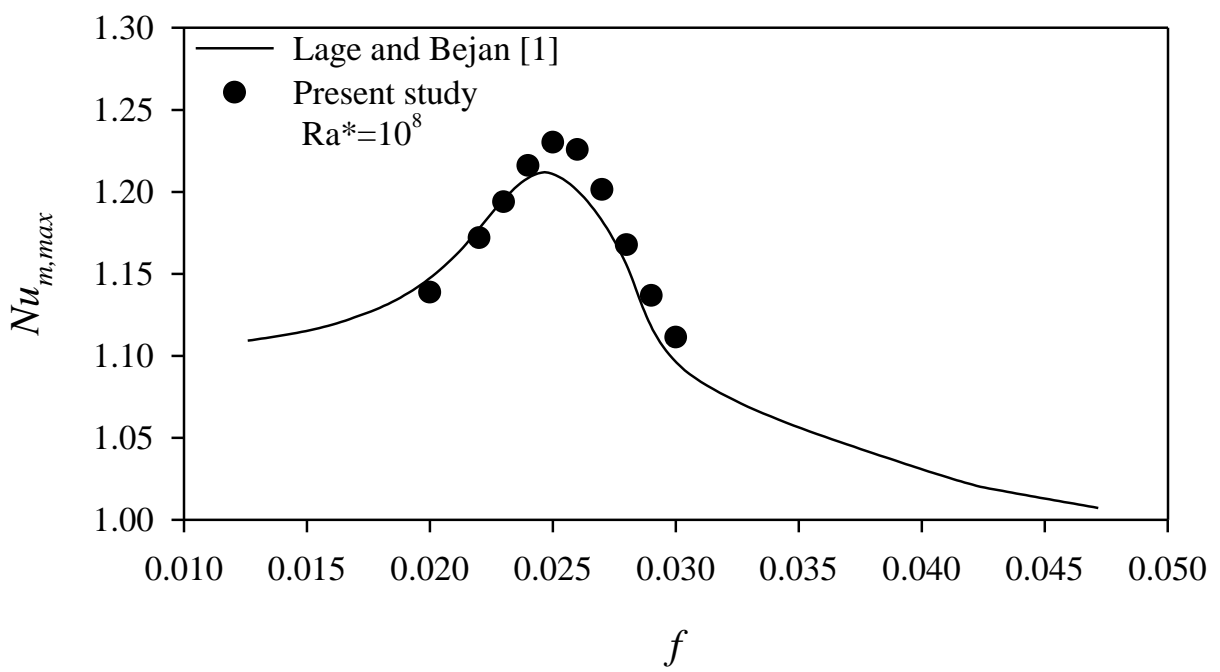

Fig. 2: The validity check of the numerical procedure.

\section{Results and Discussion}

Natural convection inside a square enclosure filled with water- $\mathrm{Al}_{2} \mathrm{O}_{3}$ nanofluid and periodically heated from one of the sidewalls is investigated numerically. The variation of the mean Nusselt number on the vertical midline of the enclosure with non-dimensional frequency, nanoparticle solid volume fractions and modified Rayleigh number values is investigated. In addition, the variation of the resonance frequency of the flow with the governing parameters is investigated. Simulations 
are carried out for three different values of $R a^{*}$ in the range of $10^{7}-10^{9}$, non-dimensionalized frequency in the range of 0.01-0.04, and five different values of the volume fraction in the range of 0.01-0.05.

The variation of the supplied dimensionless wall heat flux and $N u_{m}$ with non-dimensional time is presented in Fig. 3 for $R a^{*}=10^{7}$ and the volume fraction of 0.01 . It should be noted that the solutions are started in the steady state regime. As it can be seen from the figure, although heat flux is supplied as square wave, $N u_{m}$ shows nearly sinusoidal character. For lower values of the frequency, two peak points are observed for $N u_{m}$. A sinusoidal variation of $N u_{m}$ is observed together with an increase in the frequency.

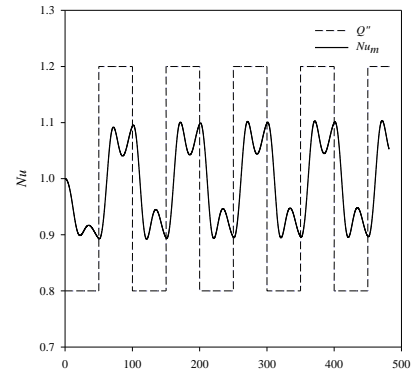

(a)

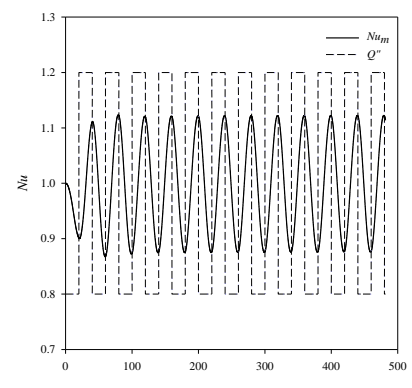

(b)

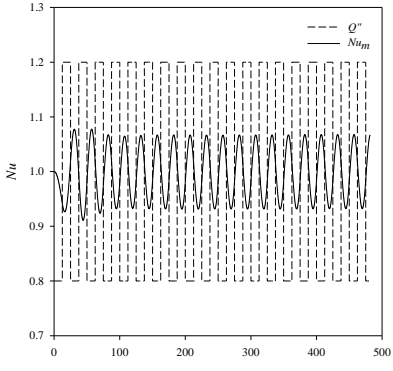

(c)

Fig. 3: Variation of supplied dimensionless wall heat flux and $\mathrm{Nu}_{\mathrm{m}}$ values with non-dimensional time for $\mathrm{Ra}^{*}=10^{7}$ : (a) $f=0.01$, (b) $f=0.025$, and (c) $f=0.04$.

In Fig. 4, the variation of the supplied dimensionless wall heat flux and $N u_{m}$ with non-dimensional time is presented for $R a^{*}=10^{9}$ and volume fraction of 0.01 . As it is seen, $N u_{m}$ variation is instable for $f=0.025$ because of the order of $R a^{*}$. A sinusoidal variation in $N u_{m}$ is again observed against the square heat flux profile for $R a^{*}=10^{9}$.

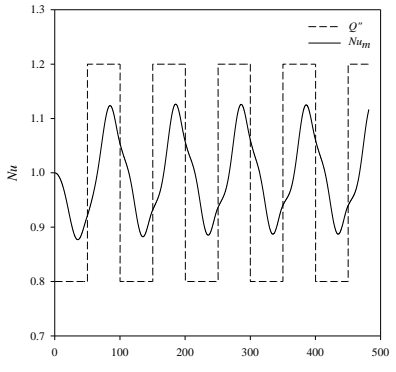

(a)

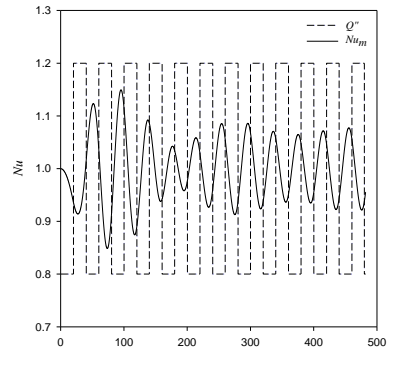

(b)

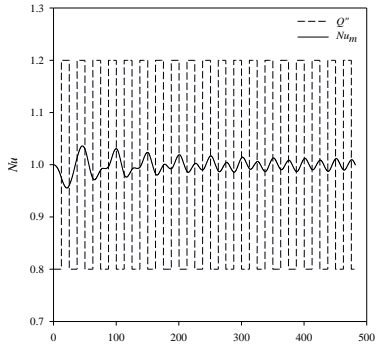

(c)

Fig. 4: The variation of the supplied dimensionless wall heat flux and $\mathrm{Nu}_{\mathrm{m}}$ values with non-dimensional time for $\mathrm{Ra}^{*}=10^{9}$ : (a) $f=0.01$, (b) $f=0.025$, and (c) $f=0.04$.

In Fig. 5, the variation of the maximum value of the mean Nusselt number on vertical midline with the solid volume fraction, the modified Rayleigh number and the dimensionless frequency is given. The resonance frequency of the flow depends both on the modified Rayleigh number and the solid volume fraction of nanoparticles. As indicated in Ref. [1], the non-dimensional resonance frequency decreases as the modified Rayleigh number increases. Heat transfer increases with the modified Rayleigh number which reduces the non-dimensional resonance frequency. In Fig. 5-a, values for $N u_{m, m a x}$ are presented for $R a^{*}=10^{7}$. Increasing solid volume fraction of nanoparticles decreases non-dimensional resonance frequency slightly. This behavior can be explained with the change of thermal properties of the fluid. In fact, an increase in nanoparticle volume fraction has the same effect as the modified Rayleigh number. Although the non-dimensional resonance frequency decreases with nanoparticle volume fraction, the $N u_{m, \max }$ values at the resonance frequencies do not change significantly with nanoparticle volume fraction. In Fig. 5-b and 5-c, $N u_{m, \max }$ values are presented for $\mathrm{Ra}^{*}=10^{8}$ and $10^{9}$, respectively. As it is clearly seen, similar changes occur at $\mathrm{Ra}^{*}=10^{8}$ and $10^{9}$. With an increase in modified Rayleigh number and volume fraction of nanoparticles decrease the non-dimensional resonance frequency. For $R a^{*}=10^{7}$, the non- 
dimensional resonance frequency decreases by $7 \%$, while the volume fraction increases from 0.01 to 0.05 . For $R a^{*}=10^{8}$ and $10^{9}$ these decreases are obtained as $8.7 \%$ and $11.1 \%$, respectively.

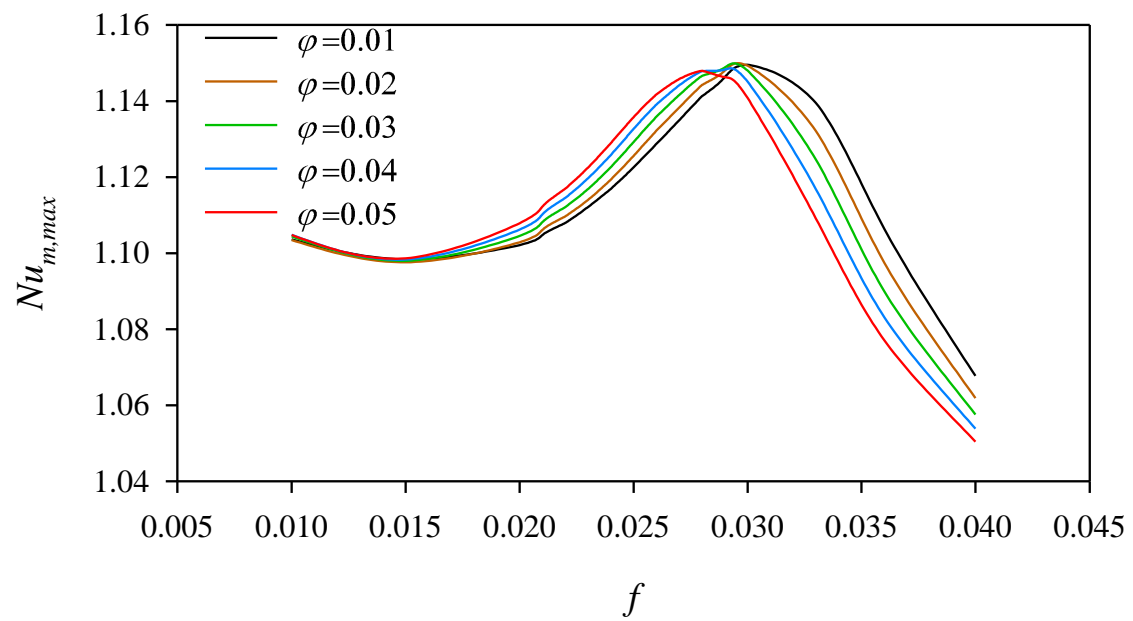

(a)

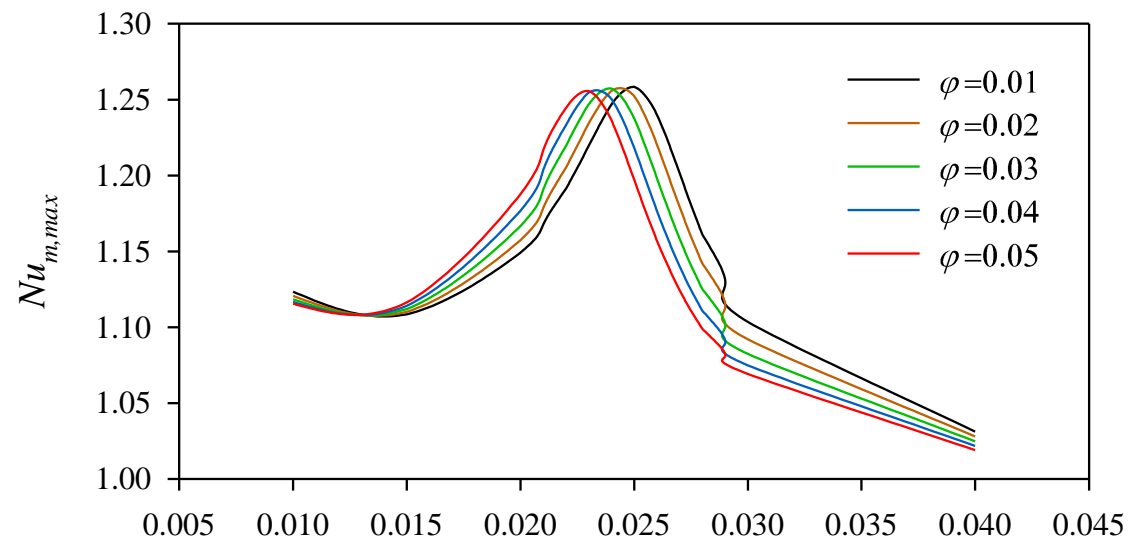

(b)

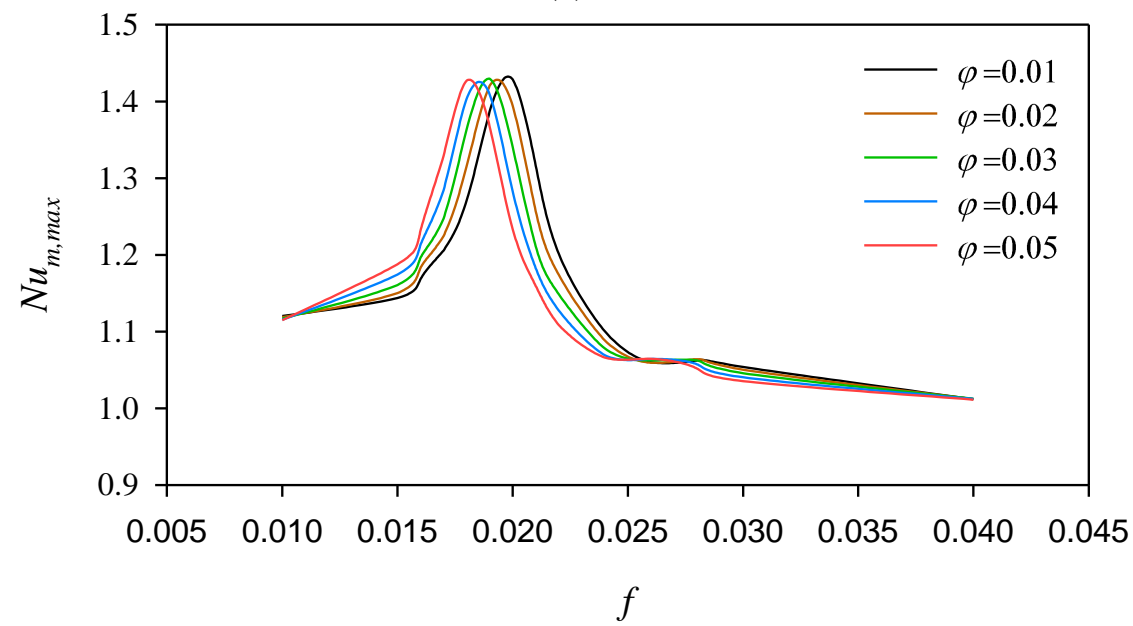

(c)

Fig. 5: The variation of $N u_{m, \max }$ with the $f$ and $R a^{*}$ : (a) $R a^{*}=10^{7}$, (b) $R a^{*}=10^{8}$, and (c) $R a^{*}=10^{9}$. 


\section{Conclusion}

Natural convection inside a square enclosure filled with nanofluid and heated periodically from the side is investigated numerically. $\mathrm{Al}_{2} \mathrm{O}_{3}$ nanoparticles-water mixture is used as the working fluid, which is modelled with the single phase model. The effects of the nanoparticle solid volume fraction on the resonance frequency is investigated and following findings are obtained:

- The resonance frequency of the flow both depends on modified Rayleigh number and solid volume fraction of nanoparticles.

- The non-dimensional resonance frequency decreases with an increase in modified Rayleigh number and an increase in solid volume fraction of nanoparticles.

- $N u_{m, \max }$ values obtained for the resonance frequencies have negligible changes with nanoparticle solid volume fraction for the same modified Rayleigh number values.

\section{References}

[1] J. Lage, A. Bejan, "The resonance of natural convection in an enclosure heated periodically from the side," International Journal of Heat and Mass Transfer, vol. 36, no. 8, pp. 2027-2038, 1993.

[2] H. S. Kwak, K. Kuwahara, J. M. Hyun, "Resonant enhancement of natural convection heat transfer in a square enclosure," International Journal of Heat and Mass Transfer, vol. 41, pp. 18, pp. 2837-2846, 1998.

[3] S. K. Kim, S. Y. Kim, Y. D. Choi, "Resonance of natural convection in a side heated enclosure with a mechanically oscillating bottom wall," International journal of heat and mass transfer, vol. 45, no. 15, pp. 3155-3162, 2002.

[4] B. Ghasemi, S. Aminossadati, "Periodic natural convection in a nanofluid-filled enclosure with oscillating heat flux," International Journal of Thermal Sciences, vol. 49, no. 1, pp. 1-9, 2010.

[5] M. Sheremet, I. Pop, O. Mahian, "Natural convection in an inclined cavity with time-periodic temperature boundary conditions using nanofluids: Application in solar collectors," International Journal of Heat and Mass Transfer, vvol. 116, pp. 751-761, 2018. 